\title{
Differential Tolerance to FTY720-Induced Antinociception in Acute Thermal and Nerve Injury Mouse Pain Models: Role of Sphingosine-1-Phosphate Receptor Adaptation $\$$
}

\author{
Laura J. Sim-Selley, Jenny L. Wilkerson, James J. Burston, ${ }^{1}$ Kurt F. Hauser, \\ Virginia McLane, Sandra P. Welch, Aron H. Lichtman, and Dana E. Selley \\ Department of Pharmacology and Toxicology, Virginia Commonwealth University, Richmond, Virginia
}

Received February 6, 2018; accepted June 19, 2018

\begin{abstract}
The immunomodulatory prodrug 2-amino-2-(2-[4-octylphenyl]ethyl)-1,3-propanediol (FTY720), which acts as an agonist for sphingosine-1-phosphate (S1P) receptors (S1PR) when phosphorylated, is proposed as a novel pain therapeutic. In this study, we assessed FTY720-mediated antinociception in the radiant heat tail-flick test and in the chronic constriction injury $(\mathrm{CCl})$ model of neuropathic pain in mice. FTY720 produced antinociception and antiallodynia, respectively, and these effects were dosedependent and mimicked by the S1PR1-selective agonist CYM5442. Repeated administration of FTY720 for 1 week produced tolerance to acute thermal antinociception, but not to antiallodynia in the $\mathrm{CCl}$ model. S1PR-stimulated $\left.{ }^{35} \mathrm{~S}\right] \mathrm{GTP} \gamma \mathrm{S}$ autoradiography revealed apparent desensitization of $G$ protein activation by S1P or the S1PR1 agonist 5-[4-phenyl-5-(trifluoromethyl)-2thienyl]-3-[3-(trifluoromethyl)phenyl]-1,2,4-oxadiazole (SEW2871) throughout the brain. Similar results were seen in spinal
\end{abstract}

cord membranes, whereby the $\mathrm{E}_{\max }$ value of S1PR-stimulated $\left[{ }^{35} \mathrm{~S}\right]$ GTP $\gamma$ S binding was greatly reduced in repeated FTY720-treated mice. These results suggest that S1PR1 is a primary target of FTY720 in alleviating both acute thermal nociception and chronic neuropathic nociception. Furthermore, the finding that tolerance develops to antinociception in the tail-flick test but not in chronic neuropathic pain suggests a differential mechanism of FTY720 action between these models. The observation that repeated FTY720 administration led to desensitized S1PR1 signaling throughout the central nervous system suggests the possibility that S1PR1 activation drives the acute thermal antinociceptive effects, whereas S1PR1 desensitization mediates the following: 1) tolerance to thermal antinociceptive actions of FTY720 and 2) the persistent antiallodynic effects of FTY720 in neuropathic pain by producing functional antagonism of pronociceptive S1PR1 signaling.

\section{Introduction}

Sphingosine-1-phosphate (S1P) is a novel pain modulatory system proposed to mediate both antinociception and pronociceptive effects depending on site of action and nociceptive test (Welch et al., 2012; Salvemini et al., 2013; Selley et al., 2013). 2-amino-2-(2-[4-octylphenyl]ethyl)-1,3-propanediol (FTY720), an immunomodulatory prodrug Food and Drug Administration approved to treat multiple sclerosis (Chun and Brinkmann, 2011), produces antiallodynic/ antihyperalgesic effects in models of inflammatory and neuropathic pain (Coste et al., 2008; Janes et al., 2014; Zhang et al., 2015). FTY720 is rapidly phosphorylated in vivo to

This work was supported by grants from the National Institutes of Health [Grant NS093990] and the Thomas F. and Kate Miller Jeffress Memorial Trust.

${ }^{1}$ Current affiliation: Arthritis Research UK Pain Centre and UK School of Life Sciences, University of Nottingham, Medical School, Queen's Medical Centre, Nottingham, United Kingdom.

https://doi.org/10.1124/jpet.118.248260.

S This article has supplemental material available at jpet.aspetjournals.org.
FTY720-phosphate (FTY720-P), which is an agonist of all S1P receptors (S1PR), except S1PR2 (Brinkmann et al., 2002).

S1P, its G protein-coupled receptors (S1PR1, S1PR2, S1PR3, and S1PR5), and synthetic enzymes (sphingosine kinases 1 and 2) are expressed throughout the central nervous system (CNS) (Edsall and Spiegel, 1999; Blondeau et al., 2007; Choi and Chun, 2013). S1P-stimulated $\left[{ }^{35} \mathrm{~S}\right] \mathrm{GTP} \gamma \mathrm{S}$ binding showed that S1PR-mediated G protein activity is distributed throughout the brain, with the highest levels in cortex, cerebellum, and amygdala (Waeber and Chiu, 1999; SimSelley et al., 2009). S1P also stimulated $\left.{ }^{35} \mathrm{~S}\right] \mathrm{GTP} \gamma \mathrm{S}$ binding in the caudate-putamen, hippocampus, hypothalamus, thalamus, and periaqueductal gray (PAG). The distribution of S1PRs is consistent with in vivo pharmacological effects of centrally administered S1P, including antinociception, hypoactivity, catalepsy, and hypothermia (Sim-Selley et al., 2009). The high level of $G$ protein activity stimulated by the S1PR1 agonist 5-[4-phenyl-5-(trifluoromethyl)-2thienyl]-3-[3-(trifluoromethyl)phenyl]-1,2,4-oxadiazole (SEW2871) and inhibition of S1P-stimulated activity by the S1PR1/3

ABBREVIATIONS: ANOVA, analysis of variance; BSA, bovine serum albumin; $\mathrm{CCl}$, chronic constriction injury; CNS, central nervous system; DMSO, dimethylsulfoxide; DRG, dorsal root ganglia; EAE, experimental autoimmune encephalomyelitis; FTY720, 2-amino-2-(2-[4-octylphenyl]ethyl)-1,3-propanediol; FTY720-P, FTY720-phosphate; i.c.v., intracerebroventricular; PAG, periaqueductal gray; ROI, region of interest; S1P, sphingosine-1-phosphate; S1PR, S1P receptor; SEW2871, 5-[4-phenyl-5-(trifluoromethyl)-2-thienyl]-3-[3-(trifluoromethyl)phenyl]-1,2,4-oxadiazole. 
antagonist VPC44116 suggest that S1PR1s are a major contributor to this activity (Sim-Selley et al., 2009).

S1PR agonists produce antinociceptive effects in multiple acute and chronic rodent pain models. Intracerebroventricular administration of S1P or SEW2871 produced thermal antinociception in mice, which was inhibited by VPC44116 (Sim-Selley et al., 2009), suggesting an antinociceptive role for central S1PR1. Likewise, intrathecal administration of dihydro-S1P decreased, whereas inhibition or genetic knockout of sphingosine kinase-2 increased, nociceptive sensitivity in the formalin and hotplate tests (Coste et al., 2008), suggesting tonic S1P activity in the spinal cord suppresses baseline sensitivity. In contrast, S1P or SEW2871 administered in the paw or dorsal root ganglia (DRG) produced increased nociceptive sensitivity in a S1PR1 antagonistreversible manner (Doyle, 2011; Xie et al., 2012), suggesting a pronociceptive role for peripheral S1PR1. Thus, S1PRs can play both anti- and pronociceptive roles, depending on their localization in specific cell types.

The observations that FTY720 and other S1PR ligands reduce nociception in chronic pain models raise the question of whether repeated agonist administration produces receptor adaptation and alters antinociceptive effects. For example, FTY720-P, but not S1P or SEW2871, downregulates S1PR1 in cell models (Gräler and Goetzl, 2004; Gonzalez-Cabrera et al., 2007; Oo et al., 2007). Similarly, mice administered repeated injections of FTY720 in the experimental autoimmune encephalomyelitis (EAE) model showed reduced S1PR1 immunoreactivity in brain (Cahalan et al., 2013). Thus, if S1PR1 activation mediates FTY720-induced antinociception, these effects might undergo tolerance following repeated administration. In contrast, FTY720 acts as a functional antagonist in the vascular system, where it downregulates S1PR1s on lymphocytes, disrupting chemotactic responses to S1P, resulting in therapeutic immunomodulation (Oo et al., 2007; Thangada et al., 2010). The role of functional antagonism of S1PR1s in neuropathic pain has recently been assessed in the rat paclitaxel model of chemotherapy-induced neuropathy (Janes et al., 2014), in which acute and repeated administration of FTY720 or other potent S1PR1 agonists reversed paclitaxel-induced mechanical allodynia and thermal hyperalgesia. These effects were mimicked by S1PR1 antagonists, but not by SEW2871. The similarity of findings with potent S1PR1 agonists and antagonists, but not with the less potent agonist SEW2871, which does not downregulate S1PR1s, suggests that functional antagonism of S1PR1s underlies the antinociceptive actions of S1PR ligands in this neuropathic pain model.

The present study investigated the consequences of FTY720 administration in acute (radiant heat tail-flick test) versus neuropathic [chronic constriction injury (CCI) of the sciatic nerve] mouse pain models. We hypothesized that distinct mechanisms mediate FTY720-induced antinociception in acute versus chronic pain. Based on the work implicating direct agonism in acute pain tests and functional antagonism in the paclitaxel neuropathic pain model, we predicted that the antinociceptive effects of repeated FTY720 administration for 1 week would be retained in the CCI model, but would undergo tolerance in the tail-flick test. We then investigated the underlying mechanism of these actions, and hypothesized that decreased S1PR-mediated G protein activation would correspond with development of tolerance to thermal antinociception and expression of antinociception in the neuropathic pain model.

\section{Materials and Methods}

Materials. S1P was purchased from Biomol International (Plymouth Meeting, PA), and 5-[4-phenyl-5-(trifluoromethyl)-2-thienyl]-3[3-(trifluoromethyl)phenyl]-1,2,4-oxadiazole (SEW2871) was purchased from Cayman Chemical (Ann Arbor, MI). FTY720 was purchased from Cayman Chemical or Tocris (Minneapolis, MN). VPC44116 was obtained from Dr. Kevin Lynch, University of Virginia (Charlottesville, VA). GDP was purchased from Sigma-Aldrich (St. Louis, MO). $\left[{ }^{35} \mathrm{~S}\right]$ GTP $\gamma \mathrm{S}$ (1250 Ci/mmol) was purchased from PerkinElmer (Boston, MA). All other reagent grade chemicals were obtained from Sigma-Aldrich or Thermo Fisher Scientific (Waltham, MA).

Subjects and Drug Treatment. Male ICR mice (Harlan Laboratories, Indianapolis, IN) weighing 25-30 g were housed in an animal care facility maintained at $22 \pm 2^{\circ} \mathrm{C}$ on a 12 -hour light/dark cycle with food and water ad libitum. All animal protocols were approved by the Virginia Commonwealth University Institutional Animal Care and Use Committee and followed the National Institutes of Health Guidelines for the Care and Use of Laboratory Animals. FTY720 was dissolved in acidified dimethylsulfoxide (DMSO) (5\% $1 \mathrm{~N} \mathrm{HCl}: 95 \%$ DMSO, vol:vol) and diluted in nine parts vehicle (1:1:18 of emulphor: ethanol:saline) to the appropriate concentration and injected via the i.p. route of administration. Mice were assessed at 15-30 minutes post-FTY720 injection because a preliminary time course showed that $6 \mathrm{mg} / \mathrm{kg}$ FTY720 (i.p.) produced maximal antinociception by $15 \mathrm{~min}-$ utes and this response remained stable for up to 1 hour, the latest time point tested. The FTY720 dose-response curve included 1, 3, 4, and $5 \mathrm{mg} / \mathrm{kg}$ FTY720 (i.p.) because pilot experiments showed minimal effect at $1 \mathrm{mg} / \mathrm{kg}$, whereas all mice reached the 10 -second cutoff at $6 \mathrm{mg} / \mathrm{kg}$. The S1PR1 antagonist VPC44116 (25 $\mu \mathrm{g})$ was prepared in acidified DMSO and infused via intracerebroventricular (i.c.v.) route of administration (Pedigo et al., 1975) 10 minutes prior to FTY720 $(10 \mathrm{mg} / \mathrm{kg}$, i.p.), and mice were tested at 25 minutes post-VPC44116 injection. Mice were assessed at 25 minutes postinjection of VPC44116 because a time point was determined at which VPC44116 produced maximal blockade of FTY720, which was when FTY720 was administered 10 minutes after VPC44116 and 15 minutes prior to testing (25 minutes after VPC44116 injection). Mice were lightly anesthetized with ether prior to i.c.v. injection; they rapidly recovered from anesthesia and were alert with no signs of distress prior to testing. CYM-5442 was injected (i.p.) 30 minutes prior to antinociceptive assessment because a pilot time course showed peak effect of this drug at 30 minutes postinjection. For repeated administration studies, mice were injected (i.p.) with $2 \mathrm{mg} / \mathrm{kg}$ FTY720 or vehicle (1:1:18 of emulphor:ethanol:saline) twice daily at 10:00 AM and 10:00 PM for 6.5 days. This dose was selected because it is approximately the $\mathrm{ED}_{50}$ for FTY720 in the tail-flick assay. Mice received only the morning injection on day 7. One group of mice was then tested for in vivo measures, and a separate group was sacrificed 24 hours later for $\left[{ }^{35} \mathrm{~S}\right]$ GTP $\gamma \mathrm{S}$ binding.

The following tests were used to determine acute CNS-mediated pharmacological effects of S1PR ligands in vivo and to assess tolerance development following repeated FTY720 administration. These tests were selected based on our earlier findings that i.c.v. administration of S1P produced thermal antinociception, hypothermia, locomotor suppression, and catalepsy (Sim-Selley et al., 2009).

Thermal Antinociception. The tail-flick test with a radiant heat stimulus was used to assess thermal nociception. Baseline latencies were determined prior to drug administration, and a 10-second cutoff time was used to prevent tail damage. Mice were not acclimated to the apparatus and were tested only for baseline and postdrug (test) latency (two trials). Antinociception was quantified as percent maximal effect $(\% \mathrm{MPE})$, where \%MPE $=[($ test - baseline $) /($ maximum baseline) $] \times 100$ for each mouse. 
Rectal Temperature. Core body temperature was measured by inserting a rectal probe connected to a Telethermometer. Baseline temperature was determined prior to drug administration, and then rectal temperature was measured after administration of FTY720 or vehicle. Values are expressed as the difference between baseline temperature and temperature after drug administration $\left(\Delta^{\circ} \mathrm{C}\right)$.

Spontaneous Activity. After temperature was measured, mice were placed in individual activity chambers, and spontaneous activity was measured for 10 minutes. Activity was measured as the total number of interruptions of 16 photocell beams per chamber during the 10-minute test period and expressed as percent inhibition of activity of the vehicle group.

Ring Immobility. After assessing spontaneous activity, mice were assessed for catalepsy in the ring immobility test in which they were placed on a ring apparatus for 5 minutes and the total time (in seconds) spent motionless was measured.

Chronic Constriction Injury Surgery. The CCI model of neuropathic pain was used to assess the antiallodynic effects of S1PR ligands. Surgical procedures and testing were conducted, as published (Kinsey et al., 2009). Mice received acetaminophen $(2.4 \mathrm{mg} / \mathrm{ml}$ in drinking water $)$ from 24 hours before surgery to 48 hours after surgery. Mice were anesthetized (1.5\% isoflurane), and the right hind leg was shaved and cleaned with Betadine and ethanol. An incision was made posterior to the femur, the muscles were separated, and the sciatic nerve was exposed and ligated twice using 5-0 braided silk sutures $1 \mathrm{~mm}$ in diameter (Surgical Specialties, Reading, PA). The muscle and skin were sutured using 4-0 nylon monofilament suture, and the mice were placed in heated cages and observed during a 2-hour postsurgical recovery period before return to the colony.

Assessment of Nociceptive Response after CCI. Nociceptive testing along with acute or repeated drug treatments began 1 week after surgery. Mice were brought into the testing room and were placed inside the testing apparatus for 60 minutes prior to drug administration. In the time course experiment, subjects received an i.p. injection of vehicle or $12 \mathrm{mg} / \mathrm{kg}$ FTY720. Mechanical allodynia was assessed with von Frey filaments (North Coast Medical, Morgan Hill, CA), using the up-down method (Chaplan et al., 1994) at 0.5, 1.5, 3, 6, and 24 hours. For FTY720 dose-response assessment, mice were tested 1.5 hours after injection, a time when peak effect of FTY720 was observed. To test the consequences of repeated FTY720 in the CCI neuropathic pain model, mice received a daily i.p. injection of vehicle $(n=5)$ or $6 \mathrm{mg} / \mathrm{kg}$ FTY720 $(n=5)$ for 6 days. On day 1, mice were tested for both mechanical and cold allodynia 1.5 hours after receiving vehicle or drug. On day 7, all mice received FTY720 $(6 \mathrm{mg} / \mathrm{kg})$ and were tested for both mechanical and cold allodynia at 1.5 hours. Intrathecal assessment of CYM-5442 was performed by the free hand method (Hylden and Wilcox, 1980) to determine the effect of this S1P1Rselective agonist on centrally mediated nociceptive responses.

The CCI surgical procedure employed elicited mechanical allodynia and cold allodynia on the ipsilateral hind paw only (Kinsey et al., 2009); therefore, the contralateral hind paw of each mouse was used as a control comparison. The plantar surface of both hind paws (CCI and ipsilateral control sides) was stimulated five times with each filament $(0.16-6.0 \mathrm{~g})$, at a frequency of approximately $2 \mathrm{~Hz}$, starting with the 0.6 -g filament and increasing until the mouse responded by clutching and/or lifting the paw off the surface of the test apparatus. Paw lifting in response to three or more stimulations was coded as a positive response. Once a positive response was detected, sequentially lower weight filaments were used to assess the sensory threshold for each paw. Following the von Frey test, $10 \mu \mathrm{l}$ acetone (99\% highperformance liquid chromatography grade; Thermo Fisher Scientific) at room temperature was projected via a $100-\mu l$ pipette (Rainin Instruments, Woburn, MA) onto the plantar surface of each hind paw (Decosterd and Woolf, 2000). Acetone was propelled from below via air burst by expressing the pipette, thereby avoiding mechanical stimulation of the paw with the pipette. Total time lifting/clutching each hind paw was recorded with an arbitrary maximum cutoff time of 20 seconds (Decosterd and Woolf, 2000).
Agonist-Stimulated $\left[{ }^{35} \mathbf{S}\right]$ GTP $\gamma \mathbf{S}$ Binding in Spinal Cord Membranes. Mice were sacrificed by decapitation, whole spinal cords were removed, and assays were conducted, as we have published (Falenski et al., 2010). Spinal cord tissue was placed in 20 vol cold membrane buffer (50 mM Tris-HCl, $3 \mathrm{mM} \mathrm{MgCl} 2,1 \mathrm{mM}$ EGTA, pH 7.4). Tissue was homogenized, centrifuged at $48,000 \mathrm{~g}$ at $4^{\circ} \mathrm{C}$ for 10 minutes, resuspended in membrane buffer, and centrifuged at $48,000 \mathrm{~g}$. Membranes were resuspended in assay buffer $(50 \mathrm{mM}$ Tris$\mathrm{HCl}, 3 \mathrm{mM} \mathrm{MgCl}, 0.2 \mathrm{mM}$ EGTA, $100 \mathrm{mM} \mathrm{NaCl}, \mathrm{pH} 7.4$ ), and protein was determined (Bradford, 1976). Concentration-effect curves were generated by incubating $10 \mu \mathrm{g}$ membrane protein in assay buffer $+0.1 \%$ bovine serum albumin (BSA) with various concentrations of $\mathrm{S} 1 \mathrm{P}, 30 \mathrm{mM}$ GDP, and $0.1 \mathrm{nM}\left[{ }^{35} \mathrm{~S}\right] \mathrm{GTP} \gamma \mathrm{S}$ in a $1 \mathrm{ml}$ total volume. Basal binding was determined without agonist, and nonspecific binding was measured with $10 \mu \mathrm{M}$ GTP $\gamma \mathrm{S}$. The reaction was terminated by rapid filtration under vacuum through Whatman GF/B glass fiber filters, followed by three washes with cold Tris buffer (50 mM Tris-HCl, $\mathrm{pH}$ 7.4). Bound radioactivity was determined by liquid scintillation spectrophotometry at $95 \%$ efficiency after overnight extraction in ScintiSafe Econo 1 scintillation fluid. All samples were assayed in triplicate, and data were analyzed as percent stimulation, as follows: (net S1P-stimulated/basal $\left[{ }^{35} \mathrm{~S}\right] \mathrm{GTP} \gamma \mathrm{S}$ binding) $\times 100 \%$.

Agonist-Stimulated $\left[{ }^{35} \mathbf{S}\right]$ GTP $\gamma$ S Autoradiography. Mice were sacrificed by rapid decapitation, and brains were removed and frozen in isopentane $\left(-30^{\circ} \mathrm{C}\right)$. Agonist-stimulated $\left[{ }^{35} \mathrm{~S}\right] \mathrm{GTP} \gamma \mathrm{S}$ autoradiography was conducted as published (Falenski et al., 2010). Triplicate coronal sections $(20 \mu \mathrm{m})$ were cut on a cryostat $\left(-20^{\circ} \mathrm{C}\right)$ and collected on gelatin-coated slides. Slides were desiccated overnight at $4^{\circ} \mathrm{C}$ and then stored at $-80^{\circ} \mathrm{C}$. For assay, slides were incubated at $25^{\circ} \mathrm{C}$ in TME buffer $\left(50 \mathrm{mM}\right.$ Tris- $\mathrm{HCl}, 3 \mathrm{mM} \mathrm{MgCl}_{2}$, $0.2 \mathrm{mM}$ EGTA, $100 \mathrm{mM} \mathrm{NaCl}$ ) for 10 minutes, then transferred to TME buffer $+0.5 \%$ BSA containing $2 \mathrm{mM}$ GDP and $10 \mathrm{mU} / \mathrm{ml}$ adenosine deaminase for 20 minutes. Sections were then incubated in $0.04 \mathrm{nM}\left[{ }^{35} \mathrm{~S}\right] \mathrm{GTP} \gamma \mathrm{S}, 2 \mathrm{mM}$ GDP, $10 \mathrm{mU} / \mathrm{ml}$ adenosine deaminase, and agonist in TME buffer + BSA, without (basal) or with maximally effective concentrations of S1P $(60 \mu \mathrm{M})$ or SEW2871 $(80 \mu \mathrm{M})$ for 2 hours at $25^{\circ} \mathrm{C}$. Slides were rinsed in Tris buffer $\left(2 \times 2\right.$ minutes at $4^{\circ} \mathrm{C}$, $50 \mathrm{mM}, \mathrm{pH} 7.4)$ and $\mathrm{ddH}_{2} \mathrm{O}\left(30\right.$ seconds, $\left.4^{\circ} \mathrm{C}\right)$, dried, and placed in cassettes with ${ }^{14} \mathrm{C}$ microscales and Kodak Biomax $\mathrm{MR}$ film for 24 hours. Films were digitized and analyzed using NIH Image. Data are reported as mean \pm S.E.M. of triplicate sections of brains from at least seven mice per group. $\left[{ }^{14} \mathrm{C}\right]$ values were corrected for $\left[{ }^{35} \mathrm{~S}\right]$, and net-stimulated $\left[{ }^{35} \mathrm{~S}\right] \mathrm{GTP} \gamma \mathrm{S}$ binding was calculated as agoniststimulated $\left[{ }^{35} \mathrm{~S}\right] \mathrm{GTP} \gamma \mathrm{S}$ binding minus basal $\left[{ }^{35} \mathrm{~S}\right] \mathrm{GTP} \gamma \mathrm{S}$ binding.

Statistical Analysis. All data are as expressed mean \pm S.E.M. In vivo dose-response curves were fit to a four-parameter model to obtain $\mathrm{ED}_{50}$ and $\mathrm{E}_{\max }$ values. Data from antagonist experiments in thermal antinociception were analyzed by analysis of variance (ANOVA) with post hoc Newman-Keuls test for multiple comparison of three groups. FTY720 dose-response curves in the CCI model were analyzed by ANOVA with post hoc Dunnett's test to compare each FTY720 dose to a fixed control condition (vehicle in ipsi- or contralateral paw). Data from the CYM-5442 (intrathecal) dose response in the CCI model were analyzed by two-way ANOVA (CYM-5442 dose $\times$ laterality) with post hoc Bonferroni's test to determine differences between ispi- and contralateral paw responses. We analyzed the von Frey (i.e., mechanical allodynia) and acetone-induced paw flinching (i.e., cold allodynia)-dependent measures using separate mixeddesign two-way ANOVAs for the time course and repeated FTY720 experiments. Injection (vehicle versus FTY720) was a between-subject factor, and time was a within-subject factor (analyzed as repeated measures), with differences between conditions at each time point determined with post hoc Bonferroni's test. For the repeated FTY720 administration injection (vehicle versus FTY720), drug was a between-subject factor and time was a within-subject factor (analyzed as repeated measures), with differences between vehicle and FTY720 treatment on each day determined with post hoc Bonferroni's test. 
Autoradiographic data were analyzed using ANOVA with post hoc Dunnett's test to determine significance of absolute stimulated $\left[{ }^{35} \mathrm{~S}\right]$ GTP $\gamma \mathrm{S}$ binding by S1P or SEW2871 compared with basal binding in each brain region of interest (ROI). Two-way ANOVA with post hoc Bonferroni's test was used to determine regions in which net SEW2871stimulated $\left[{ }^{35} \mathrm{~S}\right] \mathrm{GTP} \gamma \mathrm{S}$ binding differed from net-stimulated binding by $\mathrm{S} 1 \mathrm{P}(\mathrm{ROI} \times$ agonist $)$ and to determine the effect of repeated treatment with FTY720 versus vehicle on basal- and agonist-stimulated $\left[{ }^{35} \mathrm{~S}\right]$ GTP $\gamma$ S binding by SEW2871 or S1P as a function of brain region (ROI $\times$ treatment). Membrane homogenate $\left[{ }^{35} \mathrm{~S}\right] \mathrm{GTP} \gamma \mathrm{S}$-binding assays were first analyzed using two-way ANOVA (S1P concentration $\times$ treatment) to determine whether FTY720 treatment had a significant effect on the S1P concentration-effect curve. The curves were then fit to a one-site concentration-effect model to determine $\mathrm{E}_{\max }$ and $\mathrm{EC}_{50}$ values, and these values were compared between treatment groups using the twotailed, nonpaired Student's $t$ test. All curve-fitting and statistical analysis was performed with GraphPad Prism 5.0, and $P \leq 0.05$ was considered significant.

\section{Results}

FTY720 and S1P1-Selective Agonists Produce Thermal Antinociception in the Radiant Heat Tail-Flick Test. FTY720 (i.p.) dose-dependently produced antinociception in the tail-flick test with a $\log \mathrm{ED}_{50}$ value of $0.49 \pm 0.02(3.09 \mathrm{mg} / \mathrm{kg})$ (Fig. 1). A maximally effective dose of FTY720 (6 mg/kg, i.p.) was then examined with and without prior i.c.v. administration of VPC44116 $(25 \mu \mathrm{g})$. VPC44116 inhibited FTY720induced antinociception, although FTY720 in the presence of VPC44116 retained a slight antinociceptive effect compared to VPC44116 alone (Fig. 2A). To further test whether S1PR1 contributes to S1PR-mediated antinociception in the CNS, a second experiment was conducted using the S1PR1-selective agonist CYM-5442 (10 mg/kg, i.p.). CYM-5442 produced antinociception that was inhibited by prior i.c.v. administration of VPC44116, and the effect of CYM-5442 in the presence of this antagonist did not differ from VPC44116 alone. We next found that the prodrug VPC01091 (20 mg/kg, i.p.), which has been reported to act as a S1PR1 agonist and S1PR3 antagonist when phosphorylated in vivo (Zhu et al., 2007), produced significant antinociceptive effects in the tail-flick test that lasted for at least 1 hour (Supplemental Fig. 1). Two-way ANOVA of the time course revealed an interaction between VPC01091 and time $[\mathrm{F}(3,40)=5.70, P<0.005]$. Post hoc analysis showed a significant effect of VPC01091 at 10,30, and 60 minutes postinjection. These results indicate that thermal antinociception induced by these S1PR agonists and prodrugs was mediated at least in part by S1PR1 in the CNS.

FTY720 and a S1PR1-Selective Agonist Reverse Allodynia in the CCI Model of Neuropathic Pain. The next series of experiments assessed the antinociceptive effects of FTY720 in the CCI chronic pain model. Mice that received $\mathrm{CCI}$ and were tested 1 week postsurgery developed significant reductions in ipsilateral paw withdrawal latencies to both mechanical (von Frey filaments) and cold (acetone) stimuli compared with presurgery baseline latencies (Fig. 3). FTY720 dose-dependently reversed mechanical allodynia, with an $\mathrm{E}_{\max }$ value of $4.74 \pm 0.82 \mathrm{~g}$ and a $\log \mathrm{ED}_{50}$ value of $0.757 \pm$ $0.084(5.72 \mathrm{mg} / \mathrm{kg})$. Likewise, FTY720 dose-dependently reversed cold allodynia, with an $\mathrm{E}_{\max }$ value of $-12.99 \pm 0.67$ seconds and a $\log \mathrm{ED}_{50}$ value of $0.358 \pm 0.041(2.28 \mathrm{mg} / \mathrm{kg})$. Thus, although FTY720 was somewhat more potent to attenuate cold than mechanical allodynia, it was nonetheless effective in

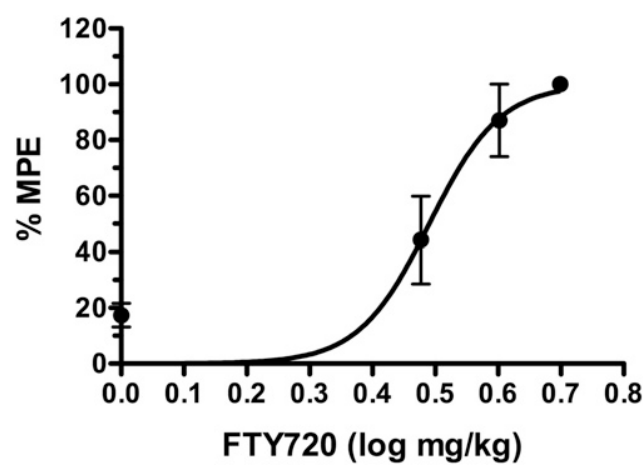

Fig. 1. FTY720 produces dose-dependent thermal antinociception in the tail-flick test. Mice were injected (i.p.) with the indicated dose of FTY720 and tested for tail-flick latency upon exposure to radiant heat 30 minutes after injection. Data are expressed as mean maximum possible effect (\% $\mathrm{MPE}) \pm$ S.E.M. $(n=5$ mice/dose $)$.

reversing both signs of CCI-induced hypersensitivity. In contrast, FTY720 did not elicit relevant effects on the paws contralateral to the CCI surgery (data not shown). The time course of effects for FTY720 (12 mg/kg) versus vehicle in the CCI model was then determined (Fig. 4). A single injection of FTY720 reversed both mechanical allodynia [interaction between treatment (FTY720 versus vehicle) and time: $\mathrm{F}(11,121)=16.5, P<$ 0.001] and cold hyperalgesia [interaction between treatment (FTY720 versus vehicle) and time: $\mathrm{F}(11,121)=61.2, P<0.001]$ from 0.5 to 6 hours. The values returned to baseline by 24 hours postinjection.

The role of S1PR1s in the spinal cord was then assessed by examining the effects of intrathecal administration of CYM5442 in CCI-induced mechanical allodynia. A preliminary time-course analysis revealed that CYM-5442 (5 $\mu \mathrm{g})$ decreased allodynia in the ipsilateral paw from 1 to 3 hours and returned to predrug thresholds by 6 hours postinjection (data not shown). One-way ANOVA showed a significant effect of time after CYM-5442 injection $[\mathrm{F}(5,24)=41.97, P<$ 0.0001], and post hoc Dunnett's test revealed significant reversal of allodynia at 1-3 hours postinjection. CYM-5442 dose-relatedly reversed mechanical allodynia in the ipsilateral paw (Fig. 5), as indicated by two-way ANOVA [interaction between paw and dose: $\mathrm{F}(2,24)=9.897, P<0.0001]$. Furthermore, one-way ANOVA revealed a significant effect of CYM-5442 dose in the ipsilateral paw $[\mathrm{F}(2,12)=15.26, P<$ $0.001]$, but not the contralateral paw $[\mathrm{F}(2,12)=1.143, P=$ 0.351]. These results indicate that S1PR ligands target S1PR1s to alleviate CCI-induced nociception.

Differential Tolerance Develops to the Antinociceptive Effects of FTY720 in the Tail-Flick Test and CCI Model of Neuropathic Pain. Studies were next conducted to determine the consequences of repeated administration of FTY720 (2 mg/kg, twice daily, i.p.) or vehicle for 6.5 days on acute thermal antinociception and other CNS-mediated effects, including locomotor inhibition, catalepsy, and hypothermia (Sim-Selley et al., 2009). On day 7 , subjects received a challenge injection of vehicle or FTY720 $(4 \mathrm{mg} / \mathrm{kg}$, i.p.). FTY720 significantly increased tail-flick latencies in repeated vehicle-treated mice, but mice treated repeatedly with FTY720 did not display antinociceptive effects (Table 1), suggesting the development of tolerance. Likewise, acute administration of FTY720 in mice treated repeatedly with vehicle displayed locomotor inhibition, catalepsy, and 


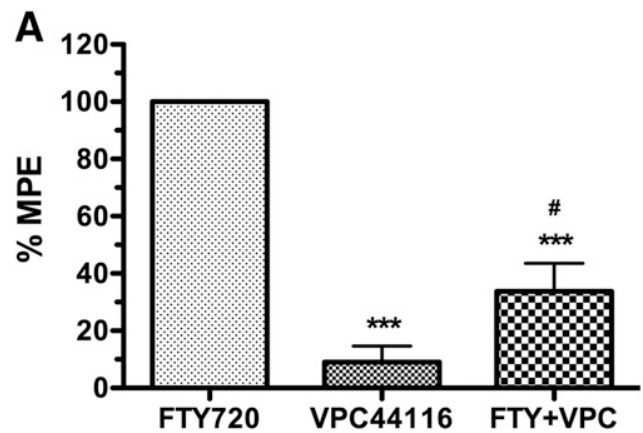

B

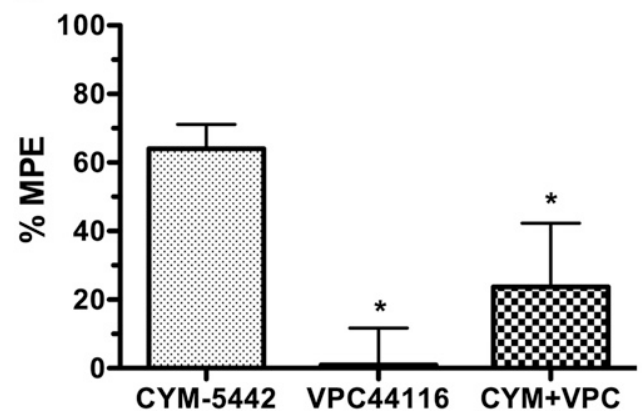

Fig. 2. The S1PR1 antagonist VPC44116 inhibits FTY720- and CYM$5442-$ mediated antinociception in the tail-flick test. Mice were injected i.c.v. with vehicle or VPC44116 $(25 \mu \mathrm{g})$, followed 10 minutes later by injection (i.p.) of FTY720 (6 mg/kg) (A) or CYM5442 (10 mg/kg) (B). Radiant heat tail-flick latencies were determined $15(\mathrm{~A})$ or $30(\mathrm{~B})$ minutes after i.p. injection. Baseline latencies were $3.97 \pm 0.26$ seconds (A) and $3.99 \pm 0.31$ seconds $(B)$. Data are expressed as mean maximum possible effect (\%MPE) \pm S.E.M. $(n=5$ mice/group). $* * * * P<0.05,0.001$ different from agonist alone; ${ }^{\#} P<0.05$ different from VPC44116 alone.

hypothermia; however, these effects underwent tolerance in mice treated repeatedly with FTY720 (Table 1).

In contrast to the antinociceptive, hypothermic, and motorsuppressive effects of FTY720, tolerance did not develop to the antiallodynic effects of FTY720 in the CCI model of neuropathic pain. After 7 days of repeated FTY720 $(6 \mathrm{mg} / \mathrm{kg})$ administration (Fig. 6A), FTY720 maintained its antiallodynic effects in both the von Frey assay of mechanical allodynia $[\mathrm{F}(1,8)=5.1, P<0.01]$ and the acetone assay of cold allodynia $[\mathrm{F}(1,8)=53.17, P<0.001$; Fig. $6 \mathrm{~B}]$.

Repeated FTY720 Treatment Desensitizes S1PRMediated G Protein Activity in the CNS. The consequences of repeated FTY720 versus vehicle administration on S1P-stimulated $G$ protein activation were examined using $\left[{ }^{35} \mathrm{~S}\right] \mathrm{GTP} \gamma \mathrm{S}$ binding in spinal cord membrane homogenates. Repeated FTY720 administration decreased S1P-stimulated $G$ protein activity compared with repeated vehicle-treated mice (Fig. 7). Two-way ANOVA revealed an interaction between repeated FTY720 administration and S1P concentration $[\mathrm{F}(5,24)=4.18, P<0.01]$. Nonlinear regression analysis of the S1P concentration-effect curves demonstrated a decrease in $\mathrm{S} 1 \mathrm{P} \mathrm{E}_{\max }$ value from $68 \% \pm 3 \%$ stimulation in vehicle-treated mice to $17 \% \pm 1 \%$ stimulation in FTY720treated mice $(P<0.0001$ by Student's $t$ test, $n=3)$, without any significant difference in $\mathrm{EC}_{50}$ values $(P=0.525, n=3)$. These results indicate apparent desensitization of S1PRmediated $\mathrm{G}$ protein activity in the spinal cord of mice treated repeatedly with FTY720.

\section{A Mechanical Allodynia}

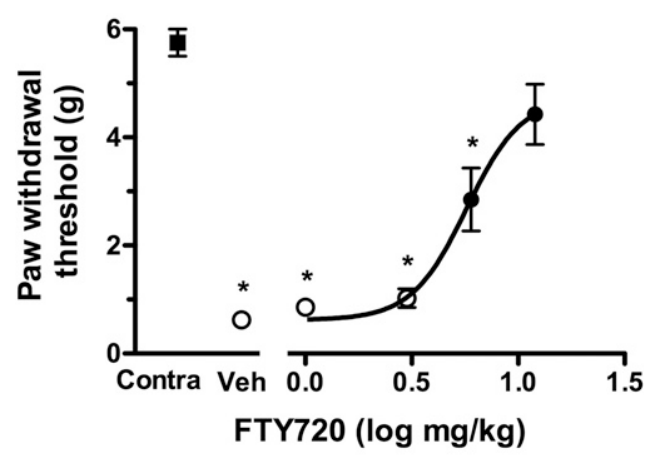

B

Cold Allodynia

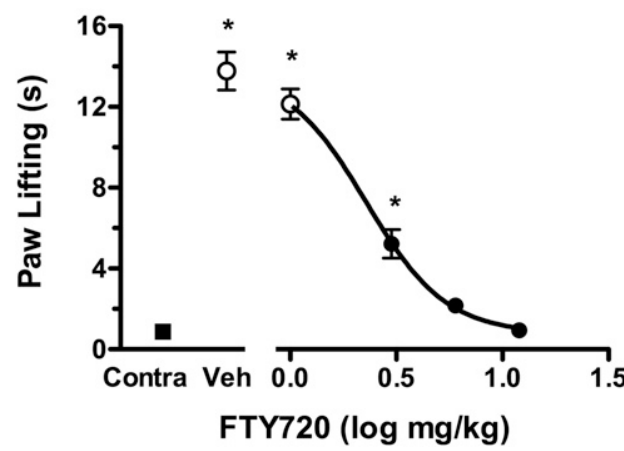

Fig. 3. FTY dose-dependently reverses mechanical (A) and cold allodynia (B) in the CCI model. Mice were injected (i.p.) with vehicle or the indicated dose of FTY720 and tested for force threshold to induce paw withdrawal in response to von Frey filament application (A) or duration of paw lifting in response to acetone application (B) to the paw contralateral (Contra) or ipsilateral to the nerve injury. Data are expressed as mean \pm S.E.M., $n=$ 8 mice/group. Contralateral data shown are from mice injected with vehicle. Closed symbols indicate that the measurement is $P<0.05$ different from the ipsilateral paw response in vehicle-injected mice. $* P<$ 0.05 different from contralateral paw response in vehicle-injected mice.

S1P- and SEW2871-stimulated $\left[{ }^{35} \mathrm{~S}\right] \mathrm{GTP} \gamma \mathrm{S}$ autoradiography was then used to determine the effect of repeated FTY720 treatment on different brain regions. The pan-S1PR endogenous agonist, S1P, and the synthetic S1PR1-selective agonist, SEW2871, stimulated $\left[{ }^{35} \mathrm{~S}\right] \mathrm{GTP} \gamma \mathrm{S}$ binding in vehicle-treated mice in the caudate-putamen, anterior cingulate cortex (Fig. 8, row 1), hippocampus, amygdala, hypothalamus (Fig. 8, row 2), PAG (Fig. 8, row 3), and cerebellum (Fig. 8, row 4). Repeated FTY-720 administration produced dramatic decreases in S1P- and SEW2871-stimulated $\left[{ }^{35} \mathrm{~S}\right] \mathrm{GTP} \gamma \mathrm{S}$ binding throughout these brain regions. In contrast, S1P-stimulated activity was still evident in the white matter of brains from FTY720treated mice (Fig. 8). In fact, the loss of high levels of S1Pstimulated activity in the gray matter highlighted the presence of remaining S1P-mediated activity in the corpus callosum (Fig. 8, row 1), stria terminalis, and fimbria (Fig. 8, row 2). Densitometric analysis was then conducted to quantify basal- and agonist-stimulated activity in repeated vehicle and FTY720-treated mice (Table 2). In vehicle-treated mice, both S1P and SEW2871 stimulated $\left.{ }^{35} \mathrm{~S}\right] \mathrm{GTP} \gamma \mathrm{S}$ binding above basal levels in all gray matter regions examined, as indicated by ANOVA with post hoc Dunnett's test in each region examined. In mice given repeated FTY720, neither S1P nor SEW2871 stimulated $\left[{ }^{35} \mathrm{~S}\right] \mathrm{GTP} \gamma \mathrm{S}$ binding significantly above 

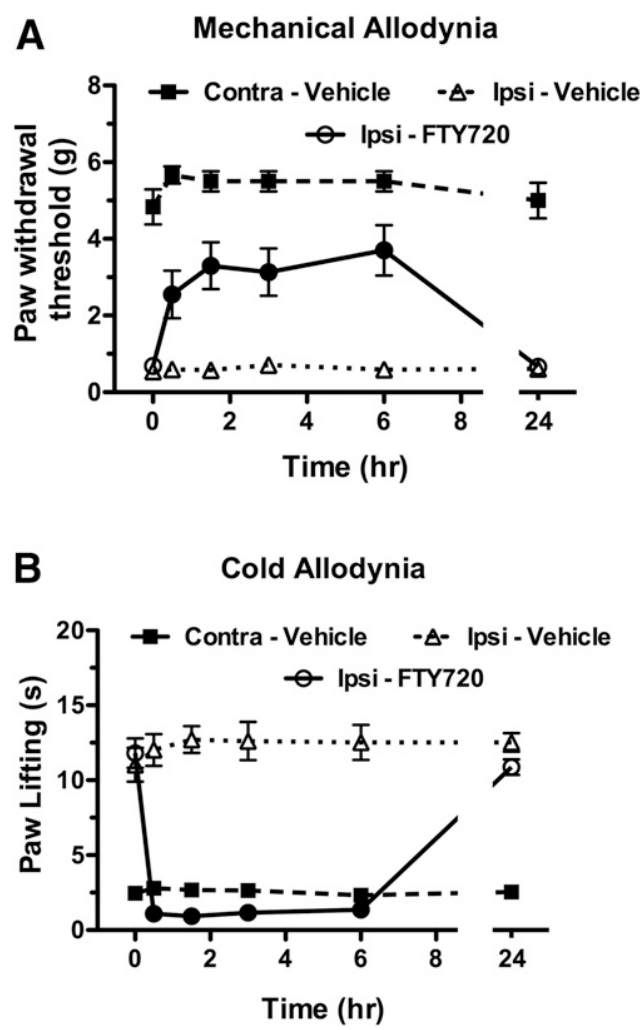

Fig. 4. FTY720 reverses allodynia for at least 6 hours in the CCI model. Mice were injected (i.p.) with vehicle or FTY720 $(12 \mathrm{mg} / \mathrm{kg})$ and tested for force threshold to induce paw withdrawal in response to von Frey filament application (A) or duration of paw lifting in response to acetone application (B) to the paw contralateral (Contra) or ipsilateral to the nerve injury. Data are expressed as mean \pm S.E.M., $n=12$ mice/group. Closed symbols indicate that the measurement is $P<0.05$ different from the ipsilateral paw response in vehicle-injected mice at the indicated time point.

basal levels in the brain nuclei examined. Interestingly, S1P produced a modest, but significant, inhibition of $\left[{ }^{35} \mathrm{~S}\right] \mathrm{GTP} \gamma \mathrm{S}$ binding below basal levels in several regions, including caudate-putamen, hippocampus, PAG, and cerebellum. S1P, but not SEW2871, also produced significant stimulation above basal levels in white matter (corpus callosum and stria terminalis) of vehicle-treated mice. Repeated FTY720 treatment reduced, but did not eliminate this activity.

To compare the effects of repeated FTY720 treatment on S1PR1- and total S1PR-mediated G protein activity as a function of brain region, data were analyzed by two-way ANOVA with region and treatment as the main factors. Analysis of S1PR1 (SEW2871)-stimulated activity showed a significant interaction between factors $[\mathrm{F}(8,116)=9.569, P<$ 0.0001]. Post hoc analysis with Bonferroni's test showed a significant decrease in every gray matter region of FTY720compared with vehicle-treated mice, but no difference in white matter (corpus callosum and stria terminalis). S1P-stimulated activity also showed a significant interaction between factors $[\mathrm{F}(8,119)=8.16, P<0.0001]$, but, in this case, post hoc analysis revealed a significant decrease in every region examined, including white matter regions. These results demonstrate profound reductions in S1PR (including S1PR1)-stimulated G protein activity in every brain region of FTY720-treated mice in which significant stimulation was observed in vehicle-treated mice.

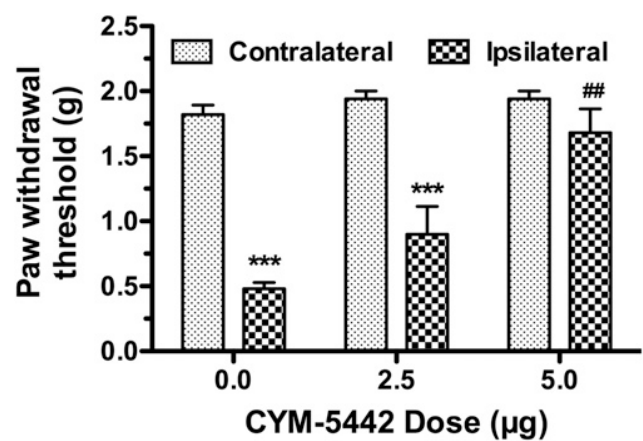

Fig. 5. Centrally administered CYM-5442 reverses allodynia in the CCI model. Mice were injected (intrathecally) with vehicle or the indicated dose of CYM-5442 and tested for force threshold to induce paw withdrawal in response to von Frey filament application to the paw contralateral or ipsilateral to the nerve injury. Data are expressed as mean \pm S.E.M., $n=$ 5 mice/group. $* * * P<0.001$ different from the response in the contralateral paw. ${ }^{\# P} P<0.01$ different from the response in the ipsilateral paw of vehicle-treated $(0 \mathrm{mg} / \mathrm{kg})$ mice.

To quantify specific S1PR-stimulated activity, net agoniststimulated $\left[{ }^{35} \mathrm{~S}\right] \mathrm{GTP} \gamma \mathrm{S}$ binding by S1P and SEW2871 was determined by subtracting basal $\left[{ }^{35} \mathrm{~S}\right] \mathrm{GTP} \gamma \mathrm{S}$ binding from total agonist-stimulated $\left.{ }^{35} \mathrm{~S}\right] \mathrm{GTP} \gamma \mathrm{S}$ binding in each ROI (Supplemental Table 1) and analyzed across regions by twoway ANOVA. Two-way ANOVA showed an interaction between agonist and region $[\mathrm{F}(8,122)=12.05, P<0.0001]$. Post hoc analysis showed that net agonist-stimulated activity did not differ between S1P and SEW2871 in most brain regions examined, suggesting that S1PR1s primarily contribute to this activity. However, SEW2871-stimulated activity was significantly lower than S1P-stimulated activity in cingulate cortex $(P<0.01)$, corpus callosum $(P<0.001)$, stria terminalis $(P<0.001)$, and caudate-putamen $(P<0.05)$ from vehicletreated mice, suggesting a potential contribution of nonS1PR1 to S1P-stimulated activity in these regions.

The consequences of repeated FTY720 administration on net S1P- and SEW2871-stimulated $\left[{ }^{35}\right.$ S $]$ GTP $\gamma$ S binding were then determined across regions (Table 2). For both S1P and SEW2871, two-way ANOVA revealed significant interactions between repeated FTY720 and brain region $[\mathrm{F}(8.120)=32.05$, $P<0.0001]$ and $[\mathrm{F}(8.117)=35.92, P<0.0001]$, respectively. Post hoc analysis with Bonferroni's test showed a significant effect of FTY720 treatment on net S1P- and SEW2871stimulated $\left[{ }^{35} \mathrm{~S}\right] \mathrm{GTP} \gamma \mathrm{S}$ binding in each region of gray matter examined. Net S1P-stimulated $\left[{ }^{35} \mathrm{~S}\right] \mathrm{GTP} \gamma \mathrm{S}$ binding was also reduced in the corpus callosum and stria terminalis by approximately 55\%-60\% compared with vehicle-treated mice. These results show that S1P- and SEW2871-stimulated $\left[{ }^{35} \mathrm{~S}\right] \mathrm{GTP} \gamma \mathrm{S}$

\section{TABLE 1}

Effects of repeated FTY720 administration on the in vivo effects of FTY720 in mice

Values are mean \pm S.E.M. of prolongation of tail-flick latency (\% maximum possible effect), net change in rectal temperature $\left({ }^{\circ} \mathrm{C}\right)$, ring immobility time (seconds), and spontaneous activity (beam breaks).

\begin{tabular}{lcccc}
\hline \multicolumn{1}{c}{ Treatment } & Tail-Flick & Temperature & Immobility & Activity \\
\hline Vehicle-Vehicle & $8 \pm 3$ & $0.0 \pm 0.3$ & $10 \pm 5$ & $404 \pm 33$ \\
Vehicle-FTY720 & $50 \pm 10^{*}$ & $-2.8 \pm 0.1^{*}$ & $72 \pm 5^{*}$ & $148 \pm 30^{*}$ \\
FTY720-FTY720 & $14 \pm 2 \S$ & $-1.1 \pm 0.2^{\S}$ & $12 \pm 2^{\S}$ & $329 \pm 30^{\S}$ \\
\hline
\end{tabular}

$* P<0.05$ different from Veh-Veh

${ }^{\S} P<0.05$ different from Veh-FTY 

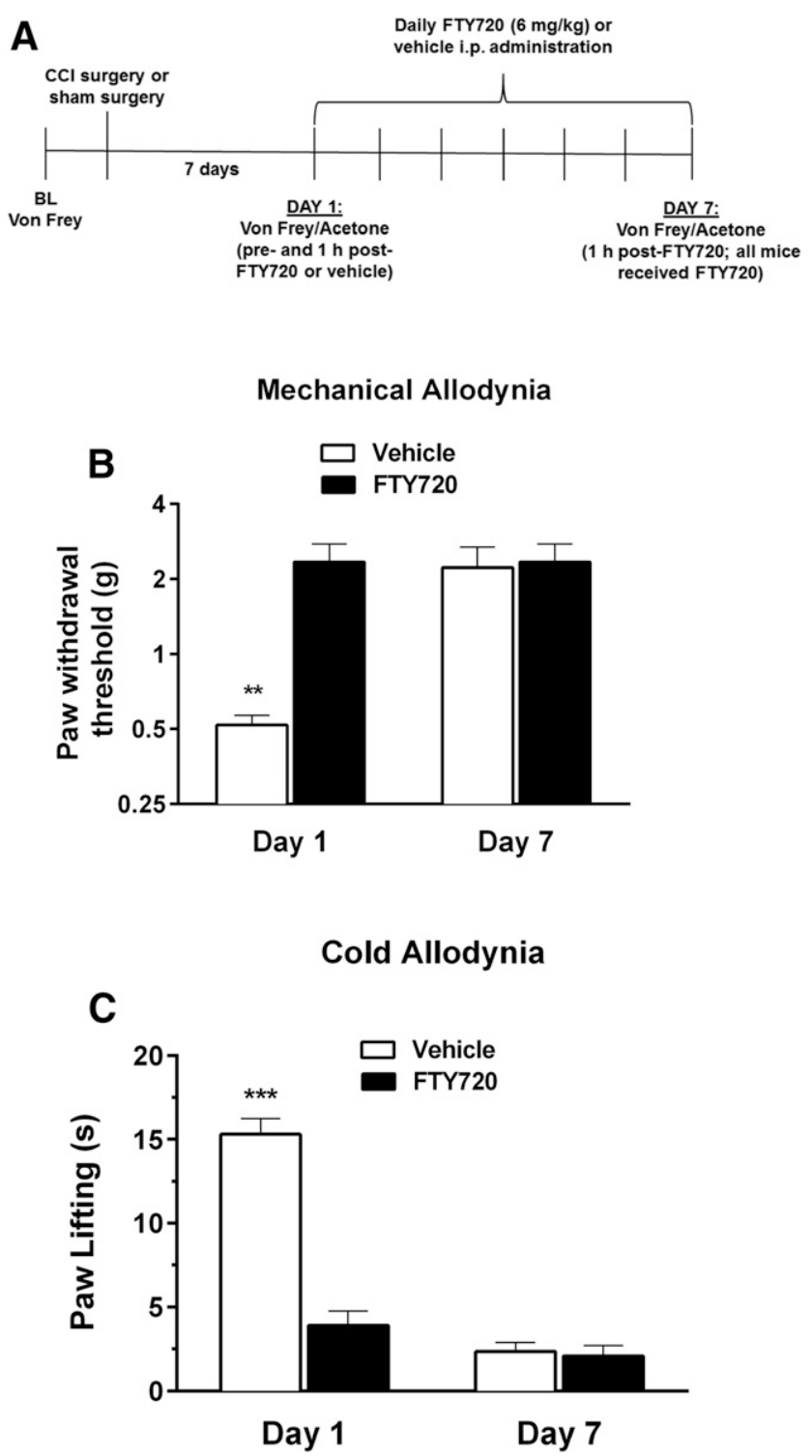

Fig. 6. Antiallodynic effects of FTY720 do not undergo tolerance. Mice were injected (i.p.) with $6 \mathrm{mg} / \mathrm{kg}$ FTY720 or vehicle once daily for 6 days, followed by $6 \mathrm{mg} / \mathrm{kg}$ FTY720 on day 7, and assessed for paw withdrawal threshold (mechanical allodynia) or acetone-induced paw flinching (cold allodynia) on days 1 and 7, as illustrated in the schematic timeline (A). FTY720 produced significant reductions in both mechanical allodynia (B) and cold allodynia (C) on days 1 and 7 compared with responses in control mice receiving vehicle on day 1 . Data are expressed as mean \pm S.E.M., $n=$ 5 mice/group. $* * P<0.01 ; * * * P<0.001$ versus all other groups.

binding were completely desensitized in gray matter areas of FTY720-treated mice, whereas S1P-stimulated $\left[{ }^{35} \mathrm{~S}\right] \mathrm{GTP} \gamma \mathrm{S}$ binding was partially desensitized in white matter.

Comparison of basal $\left.{ }^{35} \mathrm{~S}\right] \mathrm{GTP} \gamma \mathrm{S}$ binding across regions (Table 2) by two-way ANOVA revealed a main effect of region $[\mathrm{F}(8,121)=16.83, P<0.0001]$ and FTY720 treatment $[\mathrm{F}$ $(1,121)=13.58, P<0.0005]$, but no significant interaction between these factors. Basal binding data show that residual FTY720-P did not affect results because residual drug would be expected to increase basal activity. To assess the relationship between effects of FTY720 on basal $\left[{ }^{35} \mathrm{~S}\right] \mathrm{GTP} \gamma \mathrm{S}$ binding and agonist-stimulated $\left[{ }^{35} \mathrm{~S}\right] \mathrm{GTP} \gamma \mathrm{S}$ binding, linear regression analyses of normalized percentage of vehicle data were

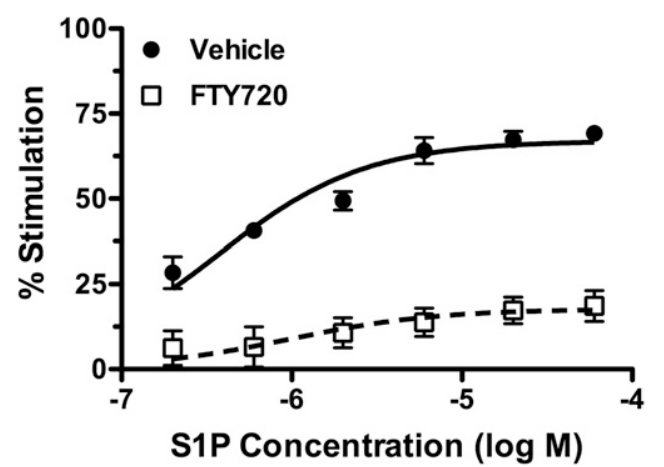

Fig. 7. S1P-stimulated $\left[{ }^{35} \mathrm{~S}\right] \mathrm{GTP} \gamma \mathrm{S}$ binding is decreased in the spinal cord of repeated FTY720-treated mice. Mice were treated with FTY720 $\left(2 \mathrm{mg} / \mathrm{kg}\right.$, twice per day) or vehicle for 6.5 days, and S1P-stimulated $\left.{ }^{35} \mathrm{~S}\right]$ GTP $\gamma$ S binding was determined using the indicated concentrations of S1P. Data are expressed as mean percentage of stimulation \pm S.E.M. $(n=$ 3 mice/group).

conducted. The effect of FTY720 was highly correlated between S1P- and SEW2871-stimulated $(r=0.953, P<0.0001)$, but not between basal- and S1P-stimulated $(r=0.356, P=$ $0.347)$ or SEW2871-stimulated $(r=0.401, P=0.284)\left[{ }^{35} \mathrm{~S}\right]$ GTP $\gamma \mathrm{S}$ binding. These results suggest that the modest reduction in basal $\left[{ }^{35} \mathrm{~S}\right] \mathrm{GTP} \gamma \mathrm{S}$ binding in FTY720-treated mice was most likely unrelated to S1PR-mediated G protein activity.

\section{Discussion}

FTY720 produced antinociception in acute thermal and chronic neuropathic pain models, strengthening support that this S1PR prodrug is analgesic across modalities. We showed repeated FTY720 administration produces tolerance to FTY720-mediated acute thermal antinociceptive, hypothermic, and locomotor depressant effects of FTY720, which was associated with S1PR desensitization. In contrast, the antiallodynic effects of FTY720 in mice receiving CCI surgery were maintained following repeated administration. Although the present results do not rule out potential development of tolerance in the CCI model following a more prolonged FTY720 dosing regimen, the S1PR regulation hypothesis of functional antagonism predicts that FTY720 potency will increase following treatment as more S1P receptors become progressively desensitized/downregulated over time. Ongoing studies are testing this hypothesis. Clinically, a differential pattern of tolerance is advantageous if reduced neuropathic pain is maintained, but side effects of FTY720 undergo tolerance. Although delineating the peripheral versus central cellular sites of FTY720 action will be critical for defining how S1PRs regulate neuropathic pain, the present finding that intrathecal CYM-5442 administration reversed mechanical allodynia is consistent with the possibility that targeting spinal S1PR1 plays a sufficient role in this neuropathic pain model.

The antinociceptive effects of FTY720 are likely mediated by S1PR1, S1PR3, and/or S1PR5 because FTY720-P does not activate S1PR2 (Brinkmann et al., 2002) and S1PR4 is not expressed in CNS (Brinkmann, 2007). Several observations support the idea that FTY720-mediated acute thermal antinociception requires S1PR1. VPC44116 (i.c.v.) antagonized thermal antinociception produced by FTY720 or CYM-5442, 
Vehicle

S1P
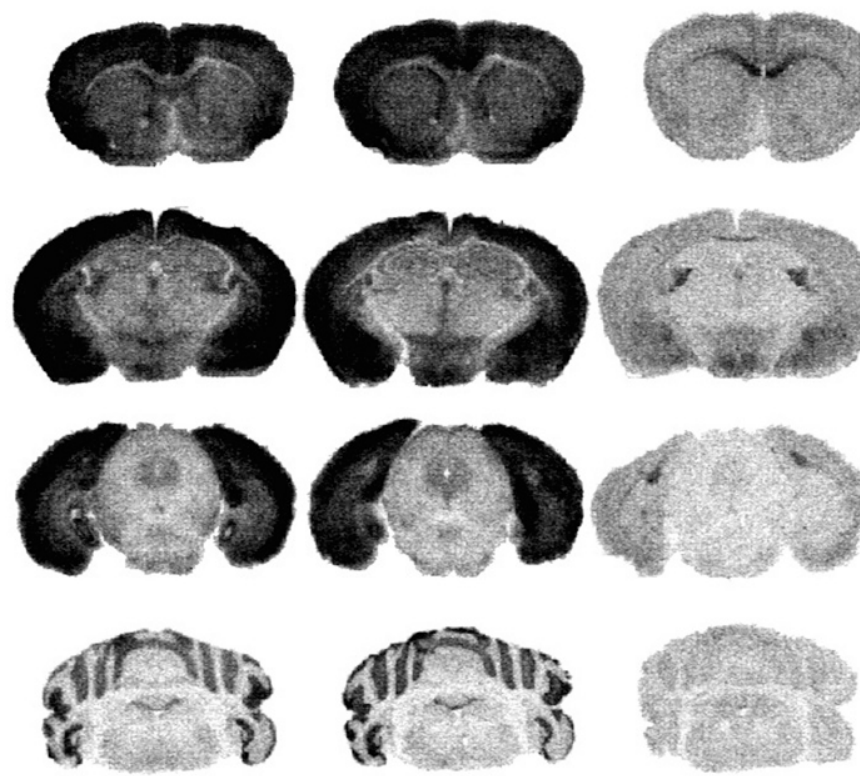

suggesting a central S1PR1 component of action. Likewise, we previously showed that S1P (i.c.v.) produced thermal antinociception that was reversed by VPC44116 and partially mimicked by SEW2871 (Sim-Selley et al., 2009). VPC44116 is only $\sim 10$-fold selective for S1PR1 versus S1PR3 (Foss et al., 2007), but the finding that FTY720-mediated antinociception was mimicked by both the S1PR1-selective agonist CYM-5442 and VPC01091, a mixed S1PR1/5 agonist/S1PR3 antagonist prodrug (Zhu et al., 2007), argues in favor of S1PR1. Moreover, FTY720-induced thermal antinociception was inhibited by an antagonist and
FTY720

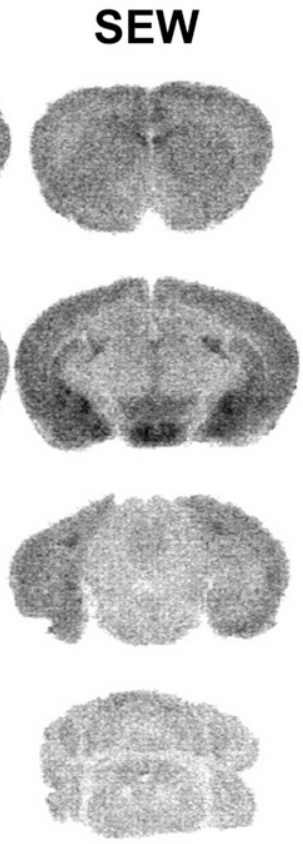

Fig. 8. Agonist-stimulated $\left[{ }^{35} \mathrm{~S}\right] \mathrm{GTP} \gamma \mathrm{S}$ autoradiography reveals widespread loss of S1PRmediated $\mathrm{G}$ protein activation in the brain in repeated FTY720-treated mice. Mice were treated with FTY720 (2 mg/kg, twice per day) or vehicle for 6.5 days, and brains were processed for S1P $(60 \mu \mathrm{M})$ - or SEW2817 $(80 \mu \mathrm{M})$-stimulated $\left[{ }^{35} \mathrm{~S}\right]$ GTP $\gamma$ S autoradiography. Representative images from mice treated with vehicle or FTY720 $(n=$ 7 to 8 mice per group) are shown in caudateputamen, cingulate cortex, and corpus callosum (row 1); hippocampus, amygdala, hypothalamus, and stria terminalis (row 2); PAG (row 3); and cerebellum (row 4).

developed tolerance upon repeated administration, indicating an agonist mechanism of action.

FTY720 dose-dependently alleviated CCI-induced mechanical and cold allodynia, an effect mimicked by intrathecal CYM-5442, suggesting a central S1PR1-mediated effect. However, the disparate effects of repeated FTY720 in CCI versus thermal antinociception suggest distinct mechanisms. Whereas FTY720-P is a S1PR agonist in thermal antinociception, it could be a functional antagonist to produce antiallodynia in CCI. Consequently, S1PRs on different target cell types might mediate FTY720 antinociception in these pain

TABLE 2

Densitometric analysis of $\left.{ }^{35} \mathrm{~S}\right] \mathrm{GTP} \gamma \mathrm{S}$ autoradiography in brains of repeated vehicle- and FTY720treated mice

Values are mean $\left[{ }^{35} \mathrm{~S}\right] \mathrm{GTP} \gamma \mathrm{S}$ bound (nCi/g) \pm S.E.M. ( $n=7$ to 8 mice per group) and were derived from densitometric analysis of the images represented in Fig. 8

\begin{tabular}{|c|c|c|c|c|}
\hline Region & Treatment & Basal $\left[{ }^{35} \mathrm{~S}\right] \mathrm{GTP} \gamma \mathrm{S}$ & $\mathrm{S} 1 \mathrm{P}\left[{ }^{35} \mathrm{~S}\right] \mathrm{GTP} \gamma \mathrm{S}$ & SEW2871 $\left[{ }^{35} \mathrm{~S}\right] \mathrm{GTP} \gamma \mathrm{S}$ \\
\hline \multirow[t]{2}{*}{ Cingulate cortex } & Vehicle & $522 \pm 29$ & $1055 \pm 42 * *$ & $942 \pm 42 * *$ \\
\hline & FTY720 & $442 \pm 32$ & $378 \pm 38 \# \#$ & $431 \pm 34^{\# \#}$ \\
\hline \multirow[t]{2}{*}{ Caudate-putamen } & Vehicle & $455 \pm 24$ & $757 \pm 38 * *$ & $663 \pm 35^{* *}$ \\
\hline & FTY720 & $433 \pm 27$ & $346 \pm 30^{\# \#}$ & $432 \pm 26^{\# \#}$ \\
\hline \multirow[t]{2}{*}{ Hippocampus } & Vehicle & $318 \pm 23$ & $619 \pm 37 * *$ & $582 \pm 47^{* *}$ \\
\hline & FTY720 & $300 \pm 21$ & $222 \pm 19 *$ & $285 \pm 26^{\# \#}$ \\
\hline \multirow[t]{2}{*}{ Amygdala } & Vehicle & $557 \pm 32$ & $1022 \pm 41^{* *}$ & $965 \pm 38 * *$ \\
\hline & FTY720 & $503 \pm 23$ & $426 \pm 33^{\# \#}$ & $476 \pm 37^{\# \#}$ \\
\hline \multirow[t]{2}{*}{ Hypothalamus } & Vehicle & $485 \pm 31$ & $714 \pm 40 * *$ & $682 \pm 42 * *$ \\
\hline & FTY720 & $428 \pm 25$ & $334 \pm 25^{*, \# \#}$ & $392 \pm 33^{\# \#}$ \\
\hline \multirow[t]{2}{*}{ PAG } & Vehicle & $417 \pm 18$ & $506 \pm 26^{*}$ & $509 \pm 23^{*}$ \\
\hline & FTY720 & $353 \pm 14$ & $259 \pm 9^{* *, \# \#}$ & $346 \pm 20 \#$ \\
\hline \multirow[t]{2}{*}{ Cerebellum } & Vehicle & $333 \pm 16$ & $508 \pm 42 * *$ & $542 \pm 37 * *$ \\
\hline & FTY720 & $274 \pm 5$ & $196 \pm 7^{* *, \# \#}$ & $266 \pm 6^{\# \#}$ \\
\hline \multirow[t]{2}{*}{ Corpus callosum } & Vehicle & $497 \pm 34$ & $866 \pm 28 * *$ & $579 \pm 29$ \\
\hline & FTY720 & $459 \pm 49$ & $625 \pm 73^{\# \#}$ & $504 \pm 59$ \\
\hline \multirow[t]{2}{*}{ Stria terminalis } & Vehicle & $473 \pm 23$ & $744 \pm 38^{* *}$ & $547 \pm 29$ \\
\hline & FTY720 & $441 \pm 35$ & $550 \pm 39^{\#}$ & $433 \pm 38$ \\
\hline
\end{tabular}

***: Agonist-stimulated values are $P<0.05,0.01$ different from the corresponding basal value in each region of vehicle- or FTY720-treated mice by ANOVA with Dunnett's post hoc test.

\#, \#\#: Values in FTY720-treated mice are $P<0.01,0.001$ different from the corresponding value in each region of vehicle-treated mice by two-way ANOVA with post hoc Bonferroni's test. 
models. We propose that an agonist action of FTY720-P at CNS neurons mediates thermal antinociception, whereas FTY720 action at spinal glia, and possibly peripheral immune cells and/or sensory neurons, mediates FTY720 effects in CCI.

FTY720-induced antinociceptive effects in CCI might be mediated by direct actions on peripheral immune cells by inhibiting $\mathrm{CD}^{+}{ }^{+} \mathrm{T}$-lymphocyte extravasation into the dorsal spinal cord (Cao and DeLeo, 2008). Alternatively, FTY720 might restrict macrophage-T lymphocyte crosstalk (Kobayashi et al., 2015), which contributes to neuropathic pain, and/or act via direct effects on neurons or glia. Glial inflammation also contributes to both lymphocyte ingress and neuropathic pain. Specifically, microglial CD40 knockdown reduced proinflammatory chemokine production and mechanical hypersensitivity in L5-transected mice (Cao et al., 2012), suggesting a role for glia-mediated spinal inflammation in neuropathic pain development. FTY720 prevents lymphocyte egress from lymph nodes (Brinkmann et al., 2004) and directly inhibits glial inflammation to improve neuronal outcomes in EAE (Groves et al., 2013). Moreover, genetic depletion of S1PR1 in astroctyes delayed the onset of mechanical hypersensitivity and diminished the antiallodynic action of FTY720 in chemotherapeutic-induced neuropathic pain (Stockstill et al., 2018). Thus, FTY720 might reduce development of CCI-induced hypersensitivity by decreasing glial inflammation and/or preventing lymphocyte extravasation.

FTY720 might also attenuate nociception in CCI by functionally antagonizing S1PRs on primary nociceptive neurons, as supported by several lines of evidence. First, S1PR1 siRNA partially inhibited mechanical hypersensitivity induced by zymosan perfusion into the DRG (Xie et al., 2012), supporting earlier findings that S1P and SEW2871 enhanced the excitability of cultured sensory neurons expressing S1PR1-4 mRNA (Chi and Nicol, 2010). Second, S1P or SEW2871 injection into the paw augmented the nociceptive effects of a TRPV1 agonist in control mice, but not in mice with genetic deletion of S1PR1 in primary nociceptive neurons (Mair et al., 2011). Third, S1PR3 predominates on DRG neurons, and its deletion reduced both $\mathrm{S} 1 \mathrm{P}$-induced nociceptor excitation and spontaneous pain behaviors (Camprubi-Robles et al., 2013), suggesting its involvement in peripheral sensitization. Nonetheless, the present finding that intrathecal administration of CYM-5442 attenuated mechanical allodynia in CCI suggests a more prominent role for S1PR1 in the spinal cord.

Agonist-stimulated $\left[{ }^{35} \mathrm{~S}\right] \mathrm{GTP} \gamma \mathrm{S}$ binding results provide further support that FTY720 behaves as an agonist at S1PR1 to produce thermal antinociception and as a functional antagonist to elicit antiallodynic effects in CCI. S1P- and SEW2871-stimulated $\left[{ }^{35} \mathrm{~S}\right] \mathrm{GTP} \gamma \mathrm{S}$ binding was profoundly desensitized in brain nuclei and spinal cord of mice receiving $4 \mathrm{mg} / \mathrm{kg}$ FTY720 per day for a week, a regimen that produced tolerance to FTY720-mediated acute thermal antinociception, hypothermia, and motor suppression, and slightly lower than the $6 \mathrm{mg} / \mathrm{kg}$ per day that did not produce tolerance to antiallodynia in CCI. Thus, the FTY720-induced decrease in S1PR1 activity corresponded positively with attenuated FTY720-mediated thermal antinociception and maintenance of FTY720-induced antiallodynia in CCI. Whether uncoupling from $\mathrm{G}$ proteins and/or downregulation of S1PR1 mediates this apparent S1PR1 desensitization remains to be determined. However, it is noteworthy that administration of $10 \mathrm{mg} / \mathrm{kg}$ per day FTY720 for 21 days in the EAE model led to significant S1PR1 downregulation in mouse brain (Cahalan et al., 2013).

As noted above, FTY720-P activates S1PR1, S1PR3, and S1PR5 in the CNS. $\left[{ }^{35} \mathrm{~S}\right] \mathrm{GTP} \gamma \mathrm{S}$ autoradiography indicated that the majority of S1PR-stimulated activity in gray matter reflected S1PR1, as indicated by the magnitude of stimulation by SEW2871 relative to $\mathrm{S} 1 \mathrm{P}$, and as previously reported (SimSelley et al., 2009). The potential contribution of S1PR3 or other S1PRs to S1P-stimulated $\left[{ }^{35} \mathrm{~S}\right] \mathrm{GTP} \gamma \mathrm{S}$ binding is difficult to ascertain. S1P stimulated somewhat greater $\mathrm{G}$ protein activity than SEW2871 in many regions, suggesting that other S1PRs might contribute to its activity and/or that S1P could have higher intrinsic efficacy than SEW2871 at S1PR1. S1PR3 also activates $\mathrm{G} \alpha$, which is not measured by $\left[{ }^{35} \mathrm{~S}\right]$ GTP $\gamma \mathrm{S}$ binding in brain, so FTY720 treatment might desensitize S1PR3-mediated $\mathrm{G} \alpha_{\mathrm{q}}$ activity not measured in this assay. The effect of FTY720 on S1PRs in gray matter differed from white matter, where S1PR5 predominates in oligodendrocytes (Jaillard et al., 2005). S1P-stimulated activity was reduced by approximately $50 \%$ in the white matter of FTY720treated brains, consistent with reports that FTY720 induces S1PR5 internalization (Gräler and Goetzl, 2004).

In comparing our findings with other recent studies, both consistencies and inconsistencies emerge with regard to agonism versus functional antagonism and the predominant role of S1PR1 in neuropathic pain. Evidence of S1PR1 functional antagonism was reported in models of chemotherapeutic-induced (paclitaxel or bortezomib) neuropathic pain (Janes et al., 2014; Stockstill et al., 2018). Acute or repeated administration of FTY720, CYM-5442, or the S1PR1 agonist ponesimod reversed chemotherapeutic-induced mechanical allodynia and thermal hyperalgesia. The S1PR1 antagonist W146 and a S1PR1 antagonist prodrug, NIBR14, mimicked this effect. However, the less potent agonist SEW2871 did not reverse paclitaxel-induced nociception, consistent with reports in cell models that FTY720 or analogs induced S1PR1 downregulation, whereas S1P or SEW2871 promoted recycling to the plasma membrane (Gräler and Goetzl, 2004; Gonzalez-Cabrera et al., 2007; Oo et al., 2007). The finding that SEW2871 does not downregulate S1PR1 suggests that functional antagonism of S1PR1 contributes to antinociception in this neuropathy model. The sustained antiallodynic actions of FTY720 in CCI following repeated administration under conditions that produce widespread desensitization of S1PR1 throughout the CNS support this hypothesis.

In contrast, S1PR1 or S1PR3 antagonists reversed FTY720 antiallodynia in the mouse spared nerve injury model of neuropathic pain (Zhang et al., 2015). However, it should be noted that a single, low dose of FTY720 $(0.01 \mathrm{mg} / \mathrm{kg})$ only partially reversed allodynia in this model, whereas higher and lower doses were ineffective. Therefore, it is possible that this effect was a direct agonist action similar to our results in thermal nociception, but the low dose was insufficient to desensitize S1PR1. In agreement with this interpretation, 16-day repeated administration of $0.01 \mathrm{mg} / \mathrm{kg}$ FTY720 did not produce tolerance when administered every other day. Thus, FTY720 may have complex and biphasic effects on neuropathic pain that are dependent on both the dose and the specific neuropathy model. Whether different cell populations mediate these effects is an important question for future investigation. 
In summary, FTY720 effectively alleviated both acute thermal and chronic neuropathic nociception in mice, via mechanisms involving S1PR1. Although acute administration of FTY720 produced antinociception in both models, repeated drug administration produced a differential tolerance. Specifically, tolerance developed to the antinociceptive effects of FTY720 in acute thermal nociception, whereas its antiallodynic effects in the CCI neuropathic pain model were maintained. These findings suggest fundamental differences in the mechanisms underlying FTY720-mediated antinociception between these two pain models, with FTY720 acting as a direct agonist in acute thermal pain and a functional antagonist in CCI-induced neuropathic pain. The profound and widespread loss of S1PR-mediated G protein activation in the CNS of repeated FTY720-treated mice supports this conclusion.

\section{Acknowledgments}

We thank Dr. Kevin R. Lynch and Dr. Timothy L. Macdonald for providing VPC44116 and VPC01091, Dr. Sarah Spiegel for helpful discussions, and Victoria Haller and David Stevens for technical assistance with the thermal antinociception experiments.

\section{Authorship Contributions}

Participated in research design: Lichtman, Selley, Sim-Selley, Welch, Wilkerson.

Conducted experiments: Sim-Selley, Welch, Wilkerson, Burston.

Performed data analysis: Lichtman, Selley, Sim-Selley, Welch,

Wilkerson, Burston.

Wrote or contributed to the writing of the manuscript: Lichtman, Selley, Sim-Selley, Welch, Wilkerson, Hauser, McLane.

\section{References}

Blondeau N, Lai Y, Tyndall S, Popolo M, Topalkara K, Pru JK, Zhang L, Kim H, Liao JK, Ding K, et al. (2007) Distribution of sphingosine kinase activity and mRNA in rodent brain. $J$ Neurochem 103:509-517.

Bradford MM (1976) A rapid and sensitive method for the quantitation of microgram quantities of protein utilizing the principle of protein-dye binding. Anal Biochem 72:248-254.

Brinkmann V (2007) Sphingosine 1-phosphate receptors in health and disease: mechanistic insights from gene deletion studies and reverse pharmacology. Pharmacol Ther 115:84-105.

Brinkmann V, Cyster JG, and Hla T (2004) FTY720: sphingosine 1-phosphate receptor-1 in the control of lymphocyte egress and endothelial barrier function. $\mathrm{Am}$ $J$ Transplant 4:1019-1025.

Brinkmann V, Davis MD, Heise CE, Albert R, Cottens S, Hof R, Bruns C, Prieschl E, Baumruker T, Hiestand P, et al. (2002) The immune modulator FTY720 targets sphingosine 1-phosphate receptors. J Biol Chem 277:21453-21457.

Cahalan SM, Gonzalez-Cabrera PJ, Nguyen N, Guerrero M, Cisar EA, Leaf NB, Brown SJ, Roberts E, and Rosen H (2013) Sphingosine 1-phosphate receptor 1 (S1P (1)) upregulation and amelioration of experimental autoimmune encephalomyelitis by an S1P(1) antagonist. Mol Pharmacol 83:316-321.

Camprubí-Robles M, Mair N, Andratsch M, Benetti C, Beroukas D, Rukwied R, Langeslag M, Proia RL, Schmelz M, Ferrer Montiel AV, et al. (2013) Sphingosine-1phosphate-induced nociceptor excitation and ongoing pain behavior in mice and humans is largely mediated by S1P3 receptor. J Neurosci 33:2582-2592.

Cao L, Beaulac H, and Eurich A (2012) Differential lumbar spinal cord responses among wild type, CD4 knockout, and CD40 knockout mice in spinal nerve L5 transection-induced neuropathic pain. Mol Pain 8:88.

Cao L and DeLeo JA (2008) CNS-infiltrating CD4+ T lymphocytes contribute to murine spinal nerve transection-induced neuropathic pain. Eur J Immunol 38: $448-458$.

Chaplan SR, Bach FW, Pogrel JW, Chung JM, and Yaksh TL (1994) Quantitative assessment of tactile allodynia in the rat paw. J Neurosci Methods 53:55-63.

Chi XX and Nicol GD (2010) The sphingosine 1-phosphate receptor, S1PR 1 , plays a prominent but not exclusive role in enhancing the excitability of sensory neurons. $J$ Neurophysiol 104:2741-2748.

Choi JW and Chun J (2013) Lysophospholipids and their receptors in the central nervous system. Biochim Biophys Acta 1831:20-32.

Chun J and Brinkmann V (2011) A mechanistically novel, first oral therapy for multiple sclerosis: the development of fingolimod (FTY720, Gilenya). Discov Med 12:213-228.

Coste O, Pierre S, Marian C, Brenneis C, Angioni C, Schmidt H, Popp L, Geisslinger G, and Scholich K (2008) Antinociceptive activity of the S1P-receptor agonist FTY720. J Cell Mol Med 12:995-1004.
Decosterd I and Woolf CJ (2000) Spared nerve injury: an animal model of persistent peripheral neuropathic pain. Pain 87:149-158.

Doyle T, Finley A, Chen Z, and Salvemini D (2011) Role for peroxynitrite in spingosine-1-phosphate-induced hyperalgesia in rats. Pain 152:643-648

Edsall LC and Spiegel S (1999) Enzymatic measurement of sphingosine 1-phosphate. Anal Biochem 272:80-86.

Falenski KW, Thorpe AJ, Schlosburg JE, Cravatt BF, Abdullah RA, Smith TH, Selley DE, Lichtman AH, and Sim-Selley LJ (2010) FAAH-/- mice display differential tolerance, dependence, and cannabinoid receptor adaptation after delta 9-tetrahydrocannabinol and anandamide administration. Neuropsychopharmacology 35:1775-1787.

Foss FW, Jr, Snyder AH, Davis MD, Rouse M, Okusa MD, Lynch KR, and Macdonald TL (2007) Synthesis and biological evaluation of gamma-aminophosphonates as potent, subtype-selective sphingosine 1-phosphate receptor agonists and antagonists. Bioorg Med Chem 15:663-677.

Gonzalez-Cabrera PJ, Hla T, and Rosen H (2007) Mapping pathways downstream of sphingosine 1-phosphate subtype 1 by differential chemical perturbation and proteomics. J Biol Chem 282:7254-7264.

Gräler MH and Goetzl EJ (2004) The immunosuppressant FTY720 down-regulates sphingosine 1-phosphate G-protein-coupled receptors. FASEB $J$ 18:551-553.

Groves A, Kihara Y, and Chun J (2013) Fingolimod: direct CNS effects of sphingosine 1-phosphate (S1P) receptor modulation and implications in multiple sclerosis therapy. $J$ Neurol Sci 328:9-18.

Hylden JL and Wilcox GL (1980) Intrathecal morphine in mice: a new technique. Eur J Pharmacol 67:313-316.

Jaillard C, Harrison S, Stankoff B, Aigrot MS, Calver AR, Duddy G, Walsh FS, Pangalos MN, Arimura N, Kaibuchi K, et al. (2005) Edg8/S1P5: an oligodendroglial receptor with dual function on process retraction and cell survival. $J$ Neurosci $\mathbf{2 5}$ : 1459-1469.

Janes K, Little JW, Li C, Bryant L, Chen C, Chen Z, Kamocki K, Doyle T, Snider A, Esposito E, et al. (2014) The development and maintenance of paclitaxel-induced neuropathic pain require activation of the sphingosine 1-phosphate receptor subtype 1. J Biol Chem 289:21082-21097.

Kinsey SG, Long JZ, O'Neal ST, Abdullah RA, Poklis JL, Boger DL, Cravatt BF, and Lichtman $\mathrm{AH}$ (2009) Blockade of endocannabinoid-degrading enzymes attenuates neuropathic pain. J Pharmacol Exp Ther 330:902-910.

Kobayashi Y, Kiguchi N, Fukazawa Y, Saika F, Maeda T, and Kishioka S (2015) Macrophage-T cell interactions mediate neuropathic pain through the glucocorticoid-induced tumor necrosis factor ligand system. J Biol Chem $\mathbf{2 9 0}$ $12603-12613$.

Mair N, Benetti C, Andratsch M, Leitner MG, Constantin CE, Camprubí-Robles M, Quarta S, Biasio W, Kuner R, Gibbins IL, et al. (2011) Genetic evidence for involvement of neuronally expressed $\mathrm{S}_{1} \mathrm{P}_{1}$ receptor in nociceptor sensitization and inflammatory pain. PLoS One 6:e17268.

Oo ML, Thangada S, Wu MT, Liu CH, Macdonald TL, Lynch KR, Lin CY, and Hla T (2007) Immunosuppressive and anti-angiogenic sphingosine 1-phosphate receptor1 agonists induce ubiquitinylation and proteasomal degradation of the receptor. $J$ Biol Chem 282:9082-9089.

Pedigo NW, Dewey WL, and Harris LS (1975) Determination and characterization of the antinociceptive activity of intraventricularly administered acetylcholine in mice. J Pharmacol Exp Ther 193:845-852.

Salvemini D, Doyle T, Kress M, and Nicol G (2013) Therapeutic targeting of the ceramide-to-sphingosine 1-phosphate pathway in pain. Trends Pharmacol Sci 34: $110-118$

Selley DE, Welch SP, and Sim-Selley LJ (2013) Sphingosine lysolipids in the CNS endogenous cannabinoid antagonists or a parallel pain modulatory system? Life Sci 93:187-193.

Sim-Selley LJ, Goforth PB, Mba MU, Macdonald TL, Lynch KR, Milstien S, Spiegel S, Satin LS, Welch SP, and Selley DE (2009) Sphingosine-1-phosphate receptors mediate neuromodulatory functions in the CNS. J Neurochem 110:1191-1202.

Stockstill K, Doyle TM, Yan X, Chen Z, Janes K, Little JW, Braden K, Lauro F, Giancotti LA, Harada CM, et al. (2018) Dysregulation of sphingolipid metabolism contributes to bortezomib-induced neuropathic pain. J Exp Med 215:1301-1313.

Thangada S, Khanna KM, Blaho VA, Oo ML, Im DS, Guo C, Lefrancois L, and Hla T (2010) Cell-surface residence of sphingosine 1-phosphate receptor 1 on lymphocytes determines lymphocyte egress kinetics. J Exp Med 207:1475-1483.

Waeber C and Chiu ML (1999) In vitro autoradiographic visualization of guanosine$5^{\prime}-O-\left(3-\left[{ }^{35} \mathrm{~S}\right]\right.$ thio)triphosphate binding stimulated by sphingosine 1-phosphate and lysophosphatidic acid. $J$ Neurochem 73:1212-1221.

Welch SP, Sim-Selley LJ, and Selley DE (2012) Sphingosine-1-phosphate receptors as emerging targets for treatment of pain. Biochem Pharmacol 84:1551-1562.

Xie W, Strong JA, Kays J, Nicol GD, and Zhang JM (2012) Knockdown of the sphingosine-1-phosphate receptor S1PR1 reduces pain behaviors induced by local inflammation of the rat sensory ganglion. Neurosci Lett 515:61-65.

Zhang DD, Linke B, Suo J, Zivkovic A, Schreiber Y, Ferreirós N, Henke M, Geisslinger G, Stark H, and Scholich K (2015) Antinociceptive effects of FTY720 during trauma-induced neuropathic pain are mediated by spinal S1P receptors. Biol Chem 396:783-794.

Zhu R, Snyder AH, Kharel Y, Schaffter L, Sun Q, Kennedy PC, Lynch KR, and Macdonald TL (2007) Asymmetric synthesis of conformationally constrained fingolimod analogues-discovery of an orally active sphingosine 1-phosphate receptor type-1 agonist and receptor type-3 antagonist. J Med Chem 50:6428-6435.

Address correspondence to: Dr. Laura J. Sim-Selley, Department of Pharmacology and Toxicology, Virginia Commonwealth University, Box 980524, 1112 East Clay Street, Richmond, Virginia 23298. E-mail: laura. sim-selley@vcuhealth.org 